\title{
Voto assimétrico, classes e mobilidade social no Brasil
}

\author{
Carlos Antonio Costa Ribeiro e Vinicius Pinheiro Israel
}

Introdução

Uma das principais questões de investigação da sociologia política é estudar a relação entre as características de estratificação social e as tendências e padrões de votação em partidos que estão competindo em eleições. Neste artigo analisamos a relação entre a estrutura de classes, os padrões de mobilidade social dos eleitores e a declaração retrospectiva de voto no segundo turno da eleição presidencial de 2006. Esse caso permite discutir a hipótese do voto assimétrico, segundo a qual a correlação entre classes sociais e voto é mediada pelos padrões de mobilidade intergeracional ascendente e descendente. Além disso, discutimos diversas outras hipóteses correlacionadas. Porém, antes é importante apresentar alguns aspectos da sociedade e da política brasileira recentes que são relevantes para entender as questões investigadas aqui.

A sociedade brasileira, embora extremamente desigual, caracteriza-se por altos níveis de mobilidade social. Entre as décadas de 1970 e 2000, o índice de mobilidade intergeracional total, que mede o percentual de pessoas em classes de destino diferentes das de origem, passou de $55,3 \%$ para $67,3 \%$, sendo que metade desse aumento se deu entre 1996 e 2008. No mesmo período, também houve uma diminuição significativa da desigualdade de renda. O coeficiente de Gini, que mede a diferença percentual entre a distribuição real e uma distribuição perfeitamente igualitária na qual cada indivíduo recebe exatamente a mesma renda, passou de 0,603 em 1996 para $0,503 \mathrm{em} 2010$. Em outras palavras, houve uma redução bastante significativa 
da desigualdade de renda e um aumento considerável da mobilidade social entre as décadas de 1990 e 2000.

Neste período também foi retomada a democracia no Brasil. Entre 1989 e o presente foram realizadas sete eleições presidenciais. Em todas houve a polarização entre o Partido dos Trabalhadores (PT) e o Partido da Reconstrução Nacional (PRN) (1989) ou o Partido da Social Democracia Brasileira (PSDB) (1994, 1998, 2002, 2006, 2010 e 2014), sendo que o PT esteve sempre mais à esquerda do que os dois outros partidos. Apesar disso, a correlação entre condições socioeconômicas dos eleitores e voto na esquerda não foi muito forte até 2006, quando parece ter havido um alinhamento entre classes trabalhadoras e voto na esquerda (PT). Singer (2012), por exemplo, sugere que houve um aumento da correlação entre classe e voto no Partido dos Trabalhadores (PT) ao longo das eleições presidenciais recentes, mas que foi na eleição de 2006 que esta correlação se tornou mais forte e evidente. A partir daí, parece ter havido uma tendência de alinhamento entre as classes trabalhadoras e o voto em Lula, candidato do PT vitorioso em 2006. As especulações de Singer foram comprovadas em um artigo recente de Limongi e Guarnieri (2015), ou seja, a eleição de 2006 parece realmente ter sido um ponto de inflexão no alinhamento entre classes sociais e voto. No entanto, é importante ter em mente que o alinhamento de 2006 não tem caráter teleológico, ou seja, segundo as teorias mais recentes sobre classe e voto, os possíveis alinhamentos eleitorais podem aumentar, diminuir ou até mesmo desaparecer ao longo de eleições sucessivas (Evans, 1996). Outro aspecto importante é a relação entre mobilidade e classes sociais. Em seu livro, Singer também sugere que a mobilidade social recente teve algum efeito sobre o alinhamento que teria ocorrido em 2006, embora não apresente análises empíricas sobre o tema.

O único trabalho que trata da correlação entre voto e mobilidade social é o de Peixoto e Rennó (2011), que analisa dados do Eseb (Estudo Eleitoral Brasileiro) de 2010 e utiliza regressões logísticas multinomiais para definir a correlação entre mobilidade social percebida e voto. Com base nessas regressões, os autores concluem que há uma associação positiva entre percepção de mobilidade social e voto em Dilma Rousseff (candidata do PT nas eleições de 2010). O principal argumento é de que o aumento da mobilidade ascendente durante os dois mandatos anteriores de Lula está correlacionado ao voto em Dilma Rousseff em 2010. Embora o trabalho seja muito interessante, há algumas limitações. Primeiro, os autores usam dados sobre mobilidade social percebida, o que obviamente não é a mesma coisa do que mobilidade social de fato. Segundo, os autores não discutem teorias sociais específicas sobre a relação entre classe, mobilidade social e voto.

Neste artigo, usamos dados sobre mobilidade social intergeracional, classes sociais e declaração de voto para analisar algumas hipóteses importantes da sociologia po- 
lítica sobre o tema. Inicialmente comparamos duas teorias mais gerais sobre voto: a teoria econômica do voto e a teoria dos grupos de referência. A primeira sugere que as condições econômicas individuais explicam os padrões de voto; a segunda, que os grupos de origem e de destino de classe social em que pessoas vivem influenciam o voto. Essas perspectivas não são antagônicas, embora as previsões sobre o efeito da mobilidade social não sejam as mesmas para ambas. Nossas análises são interessantes porque estabelecem um diálogo frutífero entre essas duas perspectivas. Além disso, buscamos discutir alguns temas importantes na área de estudos eleitorais no Brasil. Aparentemente a literatura brasileira estabeleceu uma ponte entre essas duas correntes, o que torna nosso estudo sobre mobilidade social, classes e voto uma contribuição relevante.

Além de procurar trazer algumas contribuições substantivas, nossa metodologia é relativamente inovadora nas ciências sociais brasileiras: usamos a estatística bayesiana para tomar decisões sobre as hipóteses formuladas. Essa abordagem apresenta algumas vantagens em relação à da estatística clássica: 1) trata os parâmetros como variáveis aleatórias e, com isso, consegue de forma mais natural trabalhar com os erros inerentes ao processo de inferência; 2) permite considerar estruturas não lineares e hierárquicas nos modelos; 3 ) por não utilizar o estimador de máxima verossimilhança, contorna o problema de sensibilidade numérica, de máximos locais e de maximização de funções que não são diferenciáveis; e, por fim, 4) não parte do princípio da redução do erro do tipo I (base dos testes de hipóteses clássicos), que gera viés ao ser aplicado a problemas no campo das ciências humanas. Consideramos esses aspectos fundamentais e, de certa forma, inovadores nas ciências sociais brasileiras.

O artigo está organizado em seis seções: esta introdução; apresentação das principais hipóteses que encontramos na literatura; apresentação dos dados e variáveis; descrição da metodologia e dos modelos; discussão dos resultados e avaliação das hipóteses levantadas; conclusão.

\section{Teorias e hipóteses}

A relação entre classes sociais, mobilidade intergeracional e posição política vem sendo debatida na sociologia desde o tempo dos clássicos da disciplina. Karl Marx, por exemplo, afirmava que havia uma relação direta entre classes sociais e política, mas que essa relação seria mediada pelos níveis de mobilidade social. Ao comentar a situação nos Estados Unidos, Marx (1926, p. 33) afirmou que, por causa dos altos níveis de mobilidade social naquele país, as classes ainda não teriam se formado completamente em termos de suas preferências políticas. Pelo contrário, estariam 
em "fluxo constante", ou seja, seriam compostas por pessoas que entram e saem de diferentes posições de classe e que, portanto, têm opiniões e perspectivas muito heterogêneas. Estudos mais recentes sugerem que altas taxas de mobilidade social tendem a enfraquecer a coesão de classe e podem ser um dos fatores responsáveis pelo desalinhamento de classes (Clark e Lipset, 1991).

Para entendermos melhor como as taxas absolutas de mobilidade social podem estar relacionadas com a coesão de classe, podemos usar o conceito de "identidade demográfica" sugerido por Goldthorpe (1980). De acordo com o autor, classes sociais em que a maioria das pessoas é imóvel tendem a ter mais "identidade demográfica" do que aquelas compostas por pessoas oriundas de diversas outras classes. Em nosso esquema, a classe com maior "identidade demográfica" é a de trabalhadores rurais, em que $89 \%$ dos membros são filhos de pais na mesma posição de classe, e a com menor é a classe de trabalhadores não manuais de rotina, com apenas $11 \%$ dos membros imóveis. Em outras palavras, em algumas classes as "taxas de entrada" (percentuais de pessoas em uma classe com origem nas outras classes) são altas, uma vez que muitos membros têm origens de classe distintas; enquanto em outras há muito pouca mobilidade de entrada e a maioria dos membros é imóvel intergeracionalmente. É importante levar em conta que essas especulações dizem respeito aos percentuais das classes com origens em outras classes, ou seja, dizem respeito à relação entre "taxas de entrada" e preferências políticas.

Partindo dessas ideias, podemos formular a "hipótese de composição": em classes com altas "taxas de entrada" (o que implica em pouca imobilidade), parte considerável das preferências politicas se deve ao efeito de composição (H1).

Essa primeira hipótese diz respeito aos efeitos macro ou agregados da mobilidade social. Teorias mais recentes deram espaço para hipóteses mais específicas sobre os comportamentos individuais. Na literatura sociológica contemporânea há algumas hipóteses importantes sobre o comportamento individual de voto e sua relação com classes e mobilidade social (Sobel, 1981; Clifford e Heath, 1993; De Graaf et al., 1995; Nieuwbeerta et al., 2000; Ribeiro, 2009). As hipóteses que formulamos mais adiante são relevantes porque permitem um diálogo circunstanciado de duas teorias gerais sobre voto: a "teoria econômica do voto" e a "teoria dos grupos de referência" (ou expressive theory). Segundo a primeira teoria (Downs, 1957), as pessoas votam no partido cujas políticas lhes trarão o maior benefício no futuro. Assim, o voto de classe pode ser explicado pelo fato de as pessoas em classes mais baixas terem interesses em políticas redistributivas que são tipicamente propostas por partidos de esquerda. A perspectiva da teoria dos grupos de referência ou expressive theory (Heath et al., 1985) é um pouco diferente, pois concebe o voto como um ato social em vez de um ato instrumental: "O comportamento ao votar é, portanto, a expressão de uma 
identidade política e pretende, por seu lado, refletir as normas e valores dos grupos de referência normativa do votante" (Heath et al., 1993). Os grupos aos quais as pessoas pertencem ou pertenceram no passado podem influenciar suas opiniões. Essas duas teorias não são contraditórias, mas sim complementares, tendo em vista que as pessoas podem votar em um mesmo partido seja porque compartilham o mesmo interesse, seja porque são influenciadas umas pelas outras. Ao se associar com pessoas da mesma classe, os indivíduos podem se tornar mais conscientes de seus interesses.

No entanto, essas teorias pensam a relação entre preferência política e mobilidade social de forma muito distinta. Em sua forma mais simples, a teoria econômica do voto prevê que a preferência política dos que experimentaram mobilidade social será igual à tipicamente observada na sua classe de destino - a classe de origem não teria qualquer efeito. Uma ideia simples sobre voto e mobilidade social que formulamos a partir da teoria econômica do voto - que denominamos de hipótese do efeito da classe de destino - é a seguinte: a preferência politica das pessoas que experimentaram mobilidade social estaria mais próxima da preferência típica da sua classe de destino do que daquela mais comum em sua classe de origem (H2).

A teoria dos grupos de referência, pelo contrário, prevê um efeito forte da classe de origem, uma vez que a cultura da classe de origem na juventude é provavelmente muito importante para a socialização política. No entanto, como sabemos que a mobilidade de classe se dá no máximo até os 40 anos de idade (Goldthorpe, 1980), é plausível que quanto mais velho alguém for estará mais socializado na classe de destino e mais afastado da classe de origem. Assim, pessoas mais velhas teriam preferência política mais próxima à de sua classe de destino. Em outras palavras, pessoas que experimentaram mobilidade ascendente ou descendente há pouco tempo tenderiam a ter uma posição política mais parecida com a de sua classe de origem (haveria mais aderência), ao passo que os indivíduos que já se encontram há muito tempo em uma determinada classe de destino tenderiam a estar mais "aculturados" e, portanto, a ter opinióes e votar de forma mais semelhante a outros membros de sua classe de destino. É essa perspectiva que leva à "hipótese de aculturação" (Blau, 1956) que formulamos da seguinte maneira: quanto mais velha a pessoa for menor será o efeito da classe de origem em relação ao efeito da classe de destino (H3).

Além dessa hipótese, a teoria dos grupos de referência também sugere um padrão de voto assimétrico relativo à mobilidade social. Em um estudo clássico da sociologia política, Lipset e Bendix (1959, pp. 70-71) afirmam que: “a maioria das pessoas que ascende para classe média torna-se politicamente conservadora enquanto a grande maioria dos que descendem para classe trabalhadora permanece aderente aos movimentos conservadores". Segundo essa hipótese: pessoas que tiveram mobilidade descendente permanecem votando mais de acordo com sua classe de origem mais alta 
(e mais conservadora) e pessoas que tiveram mobilidade ascendente tenderiam a se tornar mais conservadoras, na medida em que passam a adotar opiniões de sua classe de destino mais alta (e mais conservadora) (H4). No caso das eleições presidenciais de 2006, isso implicaria em dizer que pessoas com mobilidade ascendente e descendente tenderiam a declarar voto relativamente mais em Alckmin (PSDB), que estava mais à direita, do que em Lula (PT).

Embora a hipótese do voto assimétrico tenha sido formulada em termos do conservadorismo da classe média, não podemos descartar a possibilidade de haver uma assimetria oposta, ou seja, o efeito da classe de origem pode ser maior para os que têm mobilidade ascendente e menor para os que têm mobilidade descendente. Caso isso seja verificado em nossas análises, precisaremos de mais elementos para interpretar a assimetria do voto relacionada com a mobilidade social. Em 2006, Lula $(\mathrm{PT})$ era candidato à reeleição depois de ter feito um governo marcado por melhorias expressivas na economia do país. Havia um clima de governo bem-sucedido, e a popularidade do presidente era altíssima. Assim, a assimetria do voto relacionada com a mobilidade social pode estar vinculada ao voto econômico, mais especificamente às ideias de voto retrospectivo e voto prospectivo. Nesse sentido, formulamos uma última hipótese que aproxima as teorias econômica e expressiva do voto, que pode ser formulada da seguinte maneira: haveria uma assimetria em quepessoas com mobilidade ascendente tendem a votar mais de acordo com sua classe de origem (classes mais baixas votando em Lula), e neste caso estariam votando retrospectivamente em termos econômicos, na medida em que atribuem a mobilidade que tiveram ao governo anterior de Lula; e pessoas com mobilidade descendente votariam mais de acordo com sua classe de destino (classes mais baixas votando em Lula), neste caso votando prospectivamente em termos econômicos, na medida em que acreditam que as melhoras econômicas teriam continuidade com a eleição de Lula (H5).

Caso a hipótese $\mathrm{H} 3$ seja verdadeira (quanto mais velha a pessoa for, menor será o efeito da classe de origem em relação ao efeito da classe de destino), teremos evidências mais fortes para corroborar a hipótese H5. Essa interpretação é importante porque sabemos que a mobilidade ocupacional e de classe ocorre no início da carreira e, portanto, para os mais jovens. Se a assimetria prevista pela hipótese H4 for verdadeira somente para os mais jovens, o que implica também em confirmar a hipótese $\mathrm{H} 3$ de aculturação, teremos fortes evidências de que houve tendência de voto econômico prospectivo e retrospectivo relacionado com as mobilidades descendente e ascendente, respectivamente. Essa combinação das duas hipóteses implica em dizer que as teorias econômica e dos grupos de referência não podem ser fortemente separadas como sugere a literatura sobre voto e mobilidade social (por exemplo, Hout et al., 1993). 
Para discutir essas hipóteses de forma adequada, é fundamental ter dados sobre mobilidade social intergeracional, classes sociais e declaração ou intenção de voto. Por esse motivo, usamos uma pesquisa por amostra domiciliar coletada em 2008 que contém todas as informações necessárias.

\section{Dados e variáveis}

Dados

Neste artigo, usamos dados da Pesquisa Dimensões Sociais das Desigualdades (PDSD). A PDSD é uma amostra representativa da população brasileira urbana e rural, com exceção da área rural da região Norte, que inclui apenas 3,3\% da população do país. Coletada em 2008, a PDSD é composta por uma amostra de 8.048 domicílios, nos quais foram entrevistados chefes e cônjuges. A seleção dos casos seguiu um procedimento probabilístico em três etapas: na primeira foram selecionados os municípios; na segunda, os setores censitários dentro dos municípios; na terceira, os domicílios dentro dos setores. Um grande conjunto de questões, incluindo duas sobre voto na eleição presidencial de 2006, foi perguntado para chefe e cônjuge em cada domicílio. Neste artigo usamos informações para todos os entrevistados com 18 anos de idade ou mais que afirmaram ter votado em Luiz Inácio Lula da Silva ( $\mathrm{PT}$ ) ou em Geraldo Alckmin ( $\mathrm{PSDB}$ ) no segundo turno das eleições presidenciais de 2006. Assim, trabalhamos com uma amostra de 2.976 indivíduos.

\section{Variáveis e análises descritivas}

Usamos quatro variáveis: classes de origem, classes de destino, coortes de idade e declaração de voto no segundo turno da eleição presidencial de 2006, em que houve a disputa entre Luiz Inácio Lula da Silva (PT) e Geraldo Alckmin (PSDB). Há diversas formas de se definir classes sociais. Nos estudos clássicos sobre classe e voto a principal comparação é entre classe média (white-collar) e classe trabalhadora (blue-collar) (Alford, 1962). Essa distinção é, obviamente, muito simplista, porque define a estrutura de classes de maneira dicotômica. Apesar dessa limitação, vários estudos importantes foram realizados usando tabelas que cruzam voto na esquerda e na direita por classe média e classe trabalhadora (Alford, 1963; Nieuwbeerta, 1995). Uma outra alternativa é usar a educação dos eleitores para mensurar sua posição socioeconômica. Na maioria dos estudos eleitorais sobre o Brasil, essa é a única variável disponível para analisar a correlação entre condições socioeconômicas e intenções de voto (por exemplo, Carreirão, 2002; Limongi e Guarnieri, 2015). No entanto, em sociologia, a educação é frequentemente usada para medir o "capital cultural", 
que seria complementar, mas não idêntico, ao "capital econômico", que estaria mais próximo à ideia de classes sociais.

Assim, a renda seria uma alternativa mais próxima da ideia de classes sociais. No entanto, a dificuldade em usar a renda como indicador de pertencimento a alguma classe social é que frequentemente pessoas com a mesma renda podem ter interesses econômicos de longo prazo muito diferentes. Por exemplo, o operário qualificado em uma indústria e o pequeno comerciante por conta própria podem ter a mesma renda no trabalho, mas respectivamente como empregado em uma empresa e autônomo eles têm diferentes fontes de renda (provenientes de diferentes relações de emprego) e, em última instância, podem ter chances de vida muito distintas.

Uma alternativa bastante utilizada em estudos de estratificação social (Erickson e Goldthorpe, 1993; Ribeiro, 2007) e, em menor escala, em estudos de sociologia política sobre eleições e voto (Evans, 1999; Evans e De Graaf, 2013) é definir classes sociais em termos de posições ocupacionais e/ou situações de emprego. Há diversas maneiras de classificar as ocupações para definir grupos de classe. A mais usada em estudos eleitorais, no entanto, é o esquema Casmin ${ }^{1}$, que segue a ideia de que as posições na estrutura de classes são determinadas por diferentes relações de emprego e mercado (Erickson e Goldthorpe 1993). De acordo com uma longa tradição sociológica, os grupos ocupacionais ou de classe permitem definir posições distintas na estrutura produtiva das sociedades capitalistas modernas, e essas posições estariam associadas a diferentes opiniões e preferências políticas. Assim, haveria uma tendência para que pessoas em diferentes classes sociais votassem de forma distinta. As diferenças nos padrões de votação foram descritas por alguns autores como uma "luta de classes democrática" (Korpi, 1983), ou seja, as clivagens de classe na esfera política se dariam através das disputas eleitorais em que classes trabalhadoras tenderiam a votar em partidos mais à esquerda e classes médias tenderia a votar em partidos mais à direita. As disputas entre sindicatos patronais e de trabalhadores também seriam outro aspecto importante. Para analisar essa "luta de classes democrática" na esfera eleitoral usamos, neste artigo, uma versão do esquema Casmin com seis categorias: profissionais e administradores (I+II), trabalhadores não manuais de rotina (IIIab), pequenos proprietários com e sem empregados (IVab), trabalhadores manuais qualificados ( $\mathrm{V}+\mathrm{VI})$, trabalhadores manuais não qualificados (VIIa) e trabalhadores rurais e pequenos fazendeiros (IVc+VIIb).

1. Comparative Analysis of Social Mobility in Industrial Nations (Casmin) é um esquema desenvolvido por diversos pesquisadores e usado amplamente. Para uma referência de utilização, ver Erickson e Goldthorpe (1993). 
Essas classes caracterizar-se-iam por níveis altos ou baixos de especificidade nos ativos de capital humano que os indivíduos naquelas posições comandam e pela dificuldade de monitorar o trabalho desses indivíduos (Goldthorpe, 2000). Nesse esquema, a "elite" é representada por profissionais e administradores (I+II), que têm alto nível de capital humano e exercem atividade de difícil monitoramento, ou seja, têm muita liberdade para executar seu trabalho e possuem credenciais que podem ser levadas quando mudam de emprego, facilitando as possibilidades de carreira ao longo do ciclo de vida. A classe de trabalhadores não manuais de rotina (IIIab), principalmente burocratas e pessoal de escritório e vendas, conta com indivíduos com baixo capital humano e cujo trabalho é passível de algum monitoramento. Embora também sejam empregados, como a maioria dos profissionais e administradores, estão em uma posição hierarquicamente inferior nas estruturas produtivas. A classe de pequenos proprietários (IVab) é composta por pessoas que trabalham por conta própria e, portanto, têm uma situação de emprego muito diversa da dos empregados. Em geral, tendem a ser favoráveis a menos impostos e mais liberdade de mercado, o que os leva a serem relativamente menos afeitos a políticas redistributivas de esquerda. As classes de trabalhadores rurais (IVc+VIIb), trabalhadores manuais não qualificados urbanos (VIIa) e trabalhadores qualificados (V+VI) são compostas por indivíduos com pouco capital humano e cujos trabalhos são monitorados rigidamente. São as classes trabalhadoras tradicionais. O "subproletariado", categoria usada em alguns estudos brasileiros (Singer, 2012), estaria principalmente na classe de trabalhadores manuais não qualificados (VIIa), enquanto o proletariado tradicional estaria na classe de trabalhadores qualificados $(V+V I)$. É importante ressaltar que essas seis posições de classe não estão necessariamente ordenadas em termos de renda ou de educação, ou seja, esse conjunto de classes define posições em termos de relações de trabalho. Por exemplo, os pequenos proprietários (IVab) tendem a ter renda mais elevada e menos escolaridade do que os trabalhadores não manuais de rotina (IIIab). O que realmente distingue cada posição de classe são as relações de trabalho que as definem. Neste trabalho usamos o esquema com seis classes para classificar não apenas as ocupações de todas os entrevistados com 18 anos ou mais, como também a ocupação dos pais quando os respondentes tinham 14 anos de idade. As duas variáveis são cruzadas para definir uma tabela de mobilidade social intergeracional.

Para mensurar a declaração de voto, usamos as respostas a uma pergunta sobre o voto no segundo turno da eleição presidencial de 2006. Como a pesquisa foi feita em 2008, a pergunta foi retrospectiva e também incluía a possibilidade de responder que não votou, votou em branco ou que não se lembrava. De todos os respondentes com 18 anos ou mais, 67\% disseram ter votado em Lula, 13,6\%, em Alckmin, 10,2\% não votaram, 3,2\% votaram em branco ou nulo e $6,1 \%$ não se 
lembravam ou não responderam. É importante ressaltar que estamos mensurando o voto retrospectivamente e que certamente muitas pessoas que não votaram em Lula passaram a declarar que o fizeram. Na realidade, estamos medindo a percepção sobre o voto que de alguma forma é influenciada pela avaliação que os respondentes faziam do segundo governo de Lula.

Por fim, usamos uma variável para coortes de idade dividindo os respondentes em dois grupos: 18 a 40 anos e 41 ou mais anos. Tendo em vista que a mobilidade intergeracional de classes ocorre principalmente no início da carreira das pessoas (Goldthorpe, 1980, pp. 69-71), sabemos que a primeira coorte de idade, a mais nova, foi a que experimentou mobilidade social no período mais recente. As pessoas com 41 anos ou mais provavelmente experimentaram mobilidade ocupacional no início de suas carreiras, quando ainda eram mais jovens. A estrutura ocupacional ou de classe tende a ser mais fixa do que a estrutura de renda, ou seja, ao longo da vida é possível que a pessoa tenha sua renda aumentada ou diminuída, mas é muito mais difícil que troque de ocupação. Assim, a distribuição de ocupações ou de classes representa uma posição mais estrutural da estratificação das sociedades modernas.

Nas próximas seções deste artigo vamos usar essas variáveis para analisar as hipóteses sobre a relação entre classes, mobilidade social, voto e ciclo de vida derivadas das teorias que discutimos acima. Antes, no entanto, é importante observar que em nossos dados sobre a declaração de voto na eleição de 2006 encontramos uma forte correlação entre voto e classes sociais como indicado na Tabela 1.

TABELA 1

Classes por Voto no Segundo Turno das Eleições Presidenciais de 2006

\begin{tabular}{l|c|c}
\multicolumn{1}{c|}{ CLASses DE DESTINo } & LULA & ALCKMIN \\
\hline I+II - Profissionais e administradores & $70,5 \%$ & $29,5 \%$ \\
III - Trabalhadores manuais de rotina & $79,7 \%$ & $20,3 \%$ \\
IV - Pequenos proprietários, autônomos & $80,4 \%$ & $19,6 \%$ \\
V+VI - Trabalhadores manuais qualificados & $86,6 \%$ & $14,4 \%$ \\
VIIa - Trabalhadores manuais não qualificados & $86,0 \%$ & $14,0 \%$ \\
VIIb - Trabalhadores rurais & $86,9 \%$ & $13,1 \%$ \\
\hline Total & $82,5 \%$ & $17,5 \%$ \\
\hline
\end{tabular}

Fonte: PDSD (2008).

Os dados mostram que a maioria dos respondentes em todas as classes sociais declarou ter votado em Lula. O mais importante, no entanto, é observar as diferenças 
entre os percentuais. Em termos relativos, Lula (PT), candidato mais à esquerda, recebeu mais votos das classes trabalhadoras ( $\mathrm{V}+\mathrm{VI}$, viIa e viIb). O candidato mais à direita na disputa, Geraldo Alckmin (PSDB), por sua vez, recebeu relativamente mais votos entre os profissionais e administradores (I+II). Outra maneira de descrever esses números relativos é calcular as razões de chances ${ }^{2}$. Esses cálculos indicam que profissionais e administradores (I+II) tinham 1,6 vez mais chances de votar em Alckmin do que trabalhadores não manuais de rotina (III); 1,7 vez mais do que pequenos proprietários autônomos; 2,7 vezes mais do que trabalhadores manuais qualificados (V+VI); 2,6 vezes mais do que trabalhadores manuais não qualificados (vIIa); e 2,8 vezes mais do que trabalhadores rurais (virb). Cabe ainda ressaltar que, relativamente, Alckmin teve mais chances de ter votos entre trabalhadores não manuais de rotina (III) e pequenos proprietários autônomos (IV). Em outras palavras, segundo nossos dados há correlação entre voto e classes sociais.

As informações da Tabela 1, no entanto, não levam em conta os padrões de mobilidade social. Sabemos que parte das pessoas que estão em uma determinada classe social tem origem em outras classes. Ou seja, sabemos que há mobilidade social e que há, provavelmente, efeitos de composição na correlação entre classe e voto. Como dizia Marx, as "classes sociais estão em fluxo", e, portanto, as opiniões em uma determinada classe são formadas tanto por indivíduos com origem naquela classe (imóveis) quanto por pessoas com origens em outras classes (móveis).

As informações da Tabela 2 confirmam que há efeito de composição da mobilidade social e sugerem que a mobilidade intergeracional diminui a "luta de classes democrática”. Por exemplo, entre os profissionais e administradores (I+II) imóveis, $61 \%$ disseram ter votado na esquerda, em Lula, enquanto o apoio da classe como um todo foi de 70,5\%. Assim, o efeito de composição foi de $8,5 \%$. Por sua vez, na classe de trabalhadores qualificados ( $\mathrm{v}+\mathrm{vi}), 87 \%$ dos membros estáveis (imóveis) declararam ter votado em Lula, enquanto $86,6 \%$ da classe como um todo votou em Lula. No caso da classe trabalhadora, praticamente não há efeito de composição, tendo em vista que a diferença é de $0,4 \%$. Para as classes de trabalhadores não manuais de rotina (III), a diferença é de 4\%; para a classe de pequenos proprietários (IV), de $10 \%$. Em contraste, para a classe de trabalhadores manuais não qualificados (viIa), a diferença é de 3\%; para a classe de trabalhadores rurais (virb), de 1\%. Esses números indicam que há efeito de composição, mas que esse efeito se dá principalmente pela entrada nas classes I+II, III e IV de pessoas que declararam voto em Lula. Em outras

2. A razão de chances entre os eventos A e B é determinada pela fórmula $\operatorname{RC}(\mathrm{A}, \mathrm{B})=[\mathrm{P}(\mathrm{A}) \cdot(1-\mathrm{P}(\mathrm{B}))]$ / $[\mathrm{P}(\mathrm{B}) \cdot(1-\mathrm{P}(\mathrm{A}))]$, sendo A o evento "um indivíduo de uma classe vota em Alckmin" e B o evento "um indivíduo de outra classe vota em Alckmin”. 


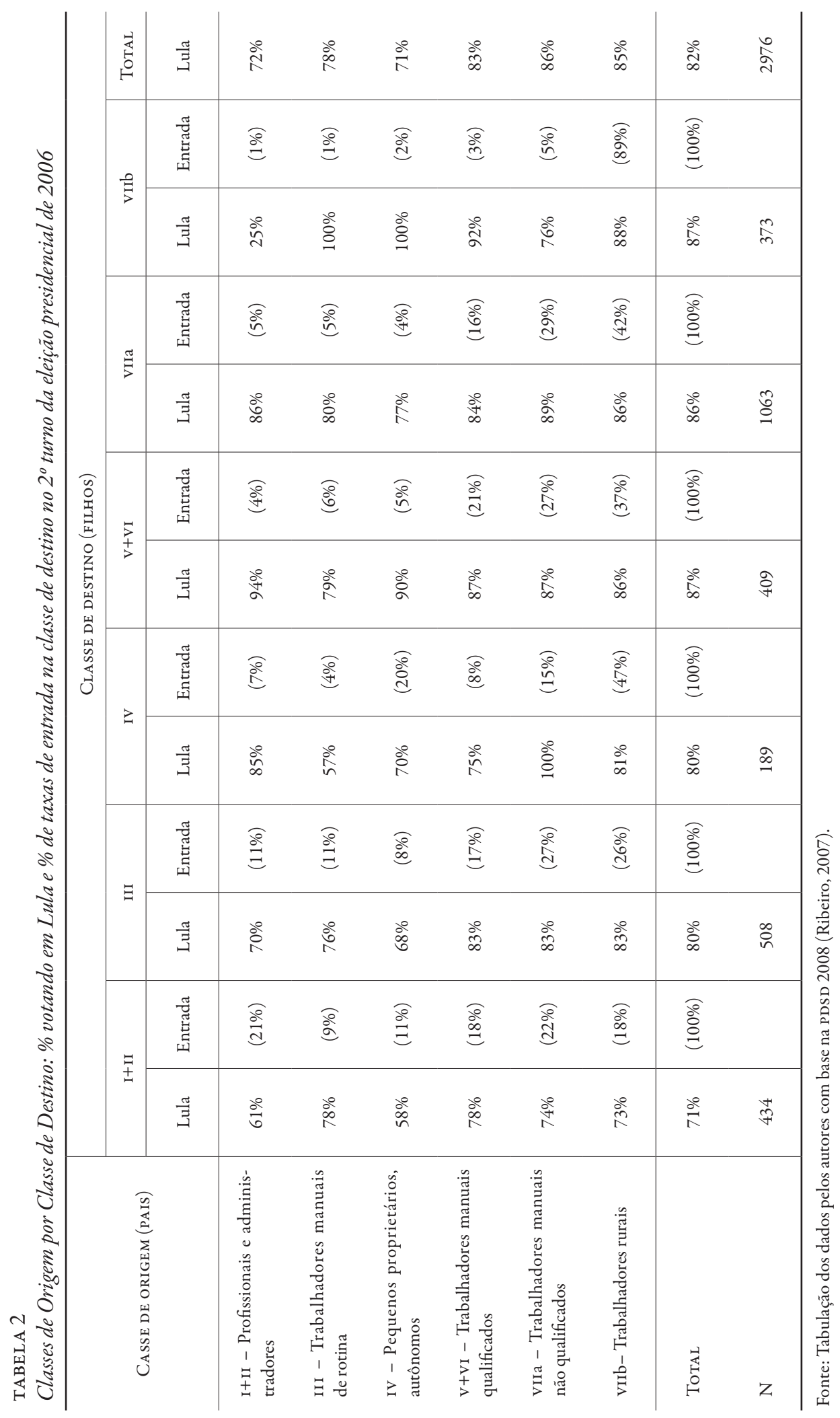


palavras, se não houvesse mobilidade nessas três classes mais altas, o percentual de pessoas votando em Lula seria menor e, consequentemente, o voto de classe seria mais acentuado.

Embora esses números mostrem claramente um efeito de composição, temos que saber se as pessoas entrando em cada classe vindas de outras classes votaram de fato mais em Lula. Ou seja, precisamos saber se as taxas de entrada eram compostas de pessoas mais à esquerda (votando mais em Lula). Por exemplo, 79\% das pessoas na classe de profissionais e administradores (I+II) têm origens em outras classes - desses novatos, $73 \%$ declararam ter votado em Lula, um número maior do que o de pessoas imóveis (61\%). Na classe de trabalhadores qualificados (v+vi), em contraste, 79\% tinham origens em outras classes e $86 \%$ dos novatos afirmaram ter votado em Lula, um número praticamente igual ao de pessoas imóveis nesta classe (87\%). Para as outras classes, os percentuais dos estáveis (imóveis) e dos novatos (móveis) votando em Lula são os seguintes: trabalhadores não manuais de rotina (III), 76\% e 62\%; pequenos proprietários (IV), 70\% e 83\%; trabalhadores manuais não qualificados (virb), 89\% e $85 \%$; e trabalhadores rurais (VIIb), $88 \%$ e $80 \%$. Em resumo, podemos dizer que o principal efeito de composição se deve à entrada de pessoas mais à esquerda nas classes I +II e IV. Se essas pessoas tivessem preferência política mais parecida com as pessoas intergeracionalmente imóveis nesses grupos, a associação entre classe e voto seria mais forte do que a observada. Em outras palavras, o fato de haver muita mobilidade social para as classes I +II e IV diminuiu a "luta de classes democrática" nas eleições presidenciais de 2006 no Brasil. Em suma, esses dados nos permitem confirmar a "hipótese de composição" para as classes I+II e IV.

\section{Metodologia}

Clifford e Heath (1993) e De Graaf et al. (1995) analisam uma série de modelos para votos categóricos binários considerando a posição dos eleitores no mercado de trabalho com base em suas localizações em tabelas de mobilidade social. Assim, os melhores modelos seriam aqueles que atribuem pesos às posições de origem e de destino de acordo com as diagonais das tabelas. As diagonais (imóveis) são locais representativos da posição política daquele grupo ocupacional ou de classe. Modelos dessa natureza, visto sob o enfoque bayesiano, podem ser tratados como modelos hierárquicos (Gelman et al., 2003; Migon et al., 2008; Gill, 2015).

O modelo de Sobel (1981) considera uma estrutura normal para a proporção encontrada nas tabelas de mobilidade. Clifford e Heath (1993) propõem assumir uma distribuição binomial para o número de indivíduos com determinada característica numa posição de mobilidade $i, j$ com indivíduos. Como a natureza dos 
dados é binomial (número de indivíduos que afirmou votar no candidato), é mais natural tratá-los seguindo essa distribuição de probabilidade. Assim, o modelo de Sobel (MS) pode ser escrito da seguinte forma:

$$
\begin{aligned}
& y_{i j} \sim \operatorname{Binomial}\left(n_{i j}, m_{i j}\right) \\
& m_{i j}=r p_{i}+(1-r) p_{j}, \quad \operatorname{com} i, j=1, \cdots, c
\end{aligned}
$$

sendo $c$ o número de posições de mobilidade, $n_{i j}$ o número de indivíduos em cada posição, $p_{i}$ a probabilidade de um indivíduo que se manteve na mesma posição de mobilidade social $i$ votar em determinado candidato e $r$ a taxa de influência da posição de origem no voto nesse candidato.

Assumindo uma extensão desse modelo que inclua uma taxa de influência da posição de origem quando há mobilidade ascendente e outra quando há mobilidade descendente, o modelo de Sobel (MS) pode ser ampliado levando ao assim chamado modelo de Sobel modificado (MSM) dado por:

$$
\begin{aligned}
& y_{i j} \sim \operatorname{Binomial}\left(n_{i j}, m_{i j}\right) \\
& m_{i j}=r_{1} p_{i}+\left(1-r_{1}\right) p_{j}, \quad \text { se } i \geq j e \\
& m_{i j}=r_{2} p_{i}+\left(1-r_{2}\right) p_{j}, \quad \text { se } i<j \operatorname{com} i, j=1, \cdots, c,
\end{aligned}
$$

no qual $r_{1}$ e $r_{2}$ são as taxas de influência da classe de origem para mobilidades ascendente e descendente, respectivamente.

Em ambos os modelos, as proporções de voto na esquerda $m_{i j}$ estão determinadas hierarquicamente pelas probabilidades da diagonal $p_{i}, i=1, \ldots, c$, e pela influência $\mathrm{da}(\mathrm{s})$ taxa(s) de origem.

Seja o vetor de parâmetros $\Theta € \Theta$, seguindo a abordagem bayesiana, a distribuição a posteriori de $\Theta$ é obtida através da fórmula de Bayes:

$$
P(\theta \mid Y)=\frac{P(Y \mid \theta) P(\theta)}{P(Y)},
$$

sendo $Y$ a matriz dos dados no qual $(Y)_{i j}=Y_{i j}{ }^{3}$

$\mathrm{Na}$ nossa abordagem, são assumidas prioris não informativas para todos os parâmetros (com distribuição uniforme no intervalo zero-um). O vetor paramétrico

3. Essa abordagem pode ser vista em Migon e Gamerman (1999) ou Gill (2015). Para uma discussão computacional, ver Gamerman e Lopes (2006). 
para o modelo MS é $\theta=\left(r, p_{P}, \ldots, p_{c}\right)$ e para o modelo modificado MSM será $\theta=\left(r_{P}\right.$ $\left.r_{2}, p_{1} . . ., p_{c}\right)$.

A verossimilhança para os modelos é da forma:

$$
P(Y \mid \theta)=\prod_{i=1}^{c} \prod_{j=1}^{c} P\left(Y_{i j} \mid \theta\right)
$$

que na escala dos logaritmos fica:

$$
L(\theta)=\ln P(Y \mid \theta)=\sum_{i=1}^{c} \sum_{j=1}^{c}\left[y_{i j} \ln \left(m_{i j}\right)+\left(n_{i j}-y_{i j}\right) \ln \left(1-m_{i j}\right)\right]
$$

Enquanto pela abordagem da estatística clássica estima-se os parâmetros maximizando a função de verossimilhança no espaço dos parâmetros $\Theta$, a estatística bayesiana trata o vetor paramétrico $\Theta$ como uma variável aleatória. As conclusões estatísticas são obtidas avaliando a distribuição a posteriori $p(\theta \mid Y)$. Os estimadores pontuais bayesianos são aqueles que minimizam as funções perda, sendo a média a posteriori, $\hat{\theta}=E_{\theta \mid Y}[\theta]$, a estatística mais utilizada (que minimiza a função perda quadrática).

Como em muitos casos não é possível obter analiticamente a densidade a posteriori $p(\theta \mid Y)$, pela intratabilidade das integrais que compõem $p(Y)$, são obtidas amostras da distribuição a posteriori de $\Theta$ pelo método de Monte Carlo via cadeia de Markov (мСмC) computados pelos programas R e Winbugs.

Resultados

A Tabela 2 de mobilidade social intergeracional (excluindo as colunas para taxas de entrada) mostra a proporção de indivíduos que afirmaram ter votado em Lula (PT) no segundo turno da eleição presidencial de 2006. Nesta seção apresentamos os ajustes de cinco modelos aos dados da Tabela 2 e a outras tabelas derivadas (diminuindo o número de classes de origem e incluindo a variável para coortes de idade). Esse exercício nos permite testar as hipóteses levantadas na segunda seção. Para cada modelo usamos duas cadeias de Markov com valores inicias diferentes, totalizando amostras com 30 mil valores. Desses, os $10 \mathrm{mil}$ primeiros, em ambas as séries, foram descartados como aquecimento (burn-in). Para evitar correlação na geração das amostras foram tomados valores de dez em dez, resultando em uma amostra a posteriori dos parâmetros de interesse para as duas séries de tamanho 2 mil.

A Tabela 3 apresenta o sumário das amostras a posteriori dos parâmetros para todos os cinco modelos estimados. A análise gráfica das amostras a posteriori e as 
medidas de convergência (não exibidas na Tabela 3) indicaram o ajuste de todos os parâmetros para os cinco modelos estimados. Os erros do MCMC não superaram $0,1 \%$ para todos os parâmetros em todos os modelos apresentados. Além disso, apresentamos na Figura 1 os box-plots das densidades a posteriori dos parâmetros estimados em cada um dos cinco modelos que usamos.

Para avaliar qual dos modelos se ajusta melhor aos dados, usamos duas estratégias. Primeiro, comparamos as estatísticas DIC - quanto menor o valor, melhor o ajuste - e, segundo, usamos o critério preditivo (Cogdon, 2005), cujos menores valores também indicam o melhor ajuste aos dados ${ }^{4}$. A medida DIC $=187,2$ indica que o Modelo 1 se ajusta bem aos dados e significa que há um efeito da mobilidade social (sem levar em conta a direção), que é significativamente diferente do efeito das classes sociais de destino. Em outras palavras, o ajuste do Modelo 1 permite concluir que não é apenas a posição de classe que está estatisticamente associada ao voto em Lula ou em Alckmin no segundo turno da eleição presidencial de 2006. A mobilidade social tem uma influência independente da classe social. No entanto, como vimos, é possível que a direção da mobilidade social esteja associada ao voto, ou seja, o efeito pode ser distinto entre a mobilidade ascendente e a descendente. Nesse sentido, ajustamos o Modelo 2, que testa a hipótese de que os efeitos das classes de origem (dos pais) são diferentes para mobilidade ascendente e descendente.

De fato, o Modelo 2 (MSM) ajusta-se melhor aos dados, tendo em vista que a estatística DIC e o critério preditivo (DIC $=184$; Preditiva $=20,8)$ são menores do que as mesmas medidas para o Modelo 1 ( DIC $=187,2$; Preditiva $=21,8)$. Os parâmetros do Modelo 2 indicam que a influência da posição de origem em mobilidade ascendente, $r_{2}$, é significativamente maior do que a influência da posição de origem quando a mobilidade é descendente, $r_{2}$. Além disso, observa-se que, conforme o índice $i$ de $p_{i}$ aumenta (representando o movimento em direção a posição de trabalhadores manuais não qualificados), as probabilidades de voto em Lula também aumentam.

O Modelo 2, no entanto, pode ser simplificado. Ao observarmos, na Tabela 3, os parâmetros estimados para a imobilidade e voto na classe de trabalhadores manuais qualificados ( $v+v i)$, trabalhadores manuais não qualificados (vira) e trabalhadores rurais (virb), é fácil perceber que o valor é praticamente o mesmo: 0,8655 (v+VI), 0,8685 (vIIa) e 0,8657 (virb) - ver também o gráfico B da Figura 1. Isso indica que

4. O critério consiste em comparar os dados observados $Y$ com um conjunto de dados $Z$ gerados por um dos modelos, condicionado aos parâmetros: $P(Z \mid Y)=\int \mathrm{P}(Z, \theta \mid Y) \mathrm{d} \theta=\int \mathrm{P}(Z \mid \theta, Y) \mathrm{P}(\theta \mid Y) \mathrm{d} \theta=\int \mathrm{P}(Z \mid \theta)$ $\mathrm{P}(\theta \mid Y) \mathrm{d} \theta$. A preditiva é obtida numericamente gerando-se da distribuição $\mathrm{P}\left(Z^{(g)} \mid \theta^{(g)}\right)$, que no nosso problema é dada por $z^{(g)} \sim \operatorname{Binomial}\left(\mathrm{m}_{i j}, \mathrm{n}_{i j}\right)$. Por fim, a medida de ajuste para cada modelo proposto é dada pelo critério $C(Z, Y)=(Z-Y)^{\prime}(Z-Y)$. Assim como o DIC, o modelo que tiver a menor medida de ajuste é considerado o melhor. 
TABELA 3

Parâmetros Estimados e Estatísticas de Ajuste para Modelos Ajustados aos Dados sobre Mobilidade Sociale Voto em Lula ou Alckmin no Segundo Turno da Eleição Presidencial de 2006

\begin{tabular}{|c|c|c|c|c|c|c|c|c|c|c|}
\hline & \multicolumn{2}{|c|}{ Modelo 1} & \multicolumn{2}{|c|}{ Modelo 2} & \multicolumn{2}{|c|}{ Modelo 3} & \multicolumn{2}{|c|}{ Modelo 4} & \multicolumn{2}{|c|}{ Modelo 5} \\
\hline & MÉDIA & DP & MÉDIA & DP & MÉDIA & $\mathrm{DP}$ & MÉDIA & DP & MÉDIA & DP \\
\hline $\mathrm{p}_{1}$ & 0,647 & 0,031 & 0,601 & 0,044 & 0,599 & 0,044 & 0,610 & 0,042 & 0,614 & 0,037 \\
\hline $\mathrm{p}_{2}$ & 0,770 & 0,029 & 0,757 & 0,033 & 0,755 & 0,033 & 0,760 & 0,032 & 0,761 & 0,031 \\
\hline $\mathrm{p}_{3}$ & 0,734 & 0,042 & 0,702 & 0,046 & 0,700 & 0,046 & 0,714 & 0,047 & 0,715 & 0,043 \\
\hline $\mathrm{P}_{4}$ & 0,863 & 0,022 & 0,866 & 0,024 & 0,867 & 0,008 & 0,868 & 0,008 & 0,869 & 0,008 \\
\hline $\mathrm{P}_{5}$ & 0,875 & 0,015 & 0,869 & 0,014 & & & & & & \\
\hline $\mathrm{P}_{6}$ & 0,868 & 0,016 & 0,866 & 0,014 & & & & & & \\
\hline$r$ & 0,469 & 0,090 & & & & & & & & \\
\hline $\mathrm{r}_{1}$ & & & 0,622 & 0,101 & 0,629 & 0,102 & & & & \\
\hline $\mathrm{r}_{2}$ & & & 0,218 & 0,128 & 0,201 & 0,123 & & & & \\
\hline$r_{11}$ & & & & & & & 0,689 & 0,136 & 0,680 & 0,132 \\
\hline$r_{21}$ & & & & & & & 0,107 & 0,097 & 0,110 & 0,101 \\
\hline$r_{12}$ & & & & & & & 0,536 & 0,136 & & \\
\hline$r_{22}$ & & & & & & & 0,473 & 0,194 & & \\
\hline $\mathrm{r}(41+\mathrm{anos})$ & & & & & & & & & 0,521 & 0,100 \\
\hline DIC & 187,15 & 184,02 & 90,13 & 156,72 & 154,94 & & & & & \\
\hline Preditiva & 21,78 & 20,85 & 49,17 & 51,89 & 48,73 & & & & & \\
\hline
\end{tabular}

não há diferença nas tendências de voto dessas três classes. Em outras palavras, não há diferença entre os três parâmetros, isto é, as distribuições a posteriori são estatisticamente as mesmas.

Diante dessas evidências combinamos as três últimas classes em um só grupo, o que nos leva a uma tabela de mobilidade com quatro classes de origem e quatro de destino. Usando essa nova tabela estimamos o Modelo 3, que testa a mesma hipótese do Modelo 2, mas combina as três classes trabalhadoras. Assim, os três últimos modelos estimados $(3,4$ e 5$)$ usam essa nova tabela com quatro classes de origem e quatro de destino, o que implica em dizer que não devem ser comparados diretamente com os dois modelos anteriores. De qualquer forma, o ajuste do Modelo 3 é muito bom $($ DIC $=90,13$ e Preditiva $=49,17)$, o que nos leva a concluir que a 
decisão de combinar as três classes trabalhadoras é estatística e substantivamente justificável.

Em suma, a comparação entre os ajustes dos Modelos 1, 2 e 3 indica que o último é o melhor, o que nos leva a concluir que os padrões de voto para os indivíduos imóveis, que permaneceram na mesma classe de seus pais, é distinto entre classes I+II, III, IV e o conjunto das classes trabalhadoras (V+VI, VIIa e viIb). Entre as três classes trabalhadoras, o padrão de voto é o mesmo, ou seja, não há distinções. Além disso, observamos, de acordo com o Modelo 3, que há um efeito assimétrico da mobilidade social, ou seja, o efeito da mobilidade ascendente é diferente do efeito da mobilidade descendente. Mais especificamente observamos que pessoas com mobilidade ascendente tendem a votar mais parecido com a posição de sua classe de origem e pessoas com mobilidade descendente tendem a votar mais de acordo com sua classe de destino. $\mathrm{O}$ ajuste desse modelo indica que a hipótese 4 está correta.

FIGURA 1

Box-plots das Densidades a posteriori dos Parâmetros Estimados pelos Cinco Modelos Apresentados
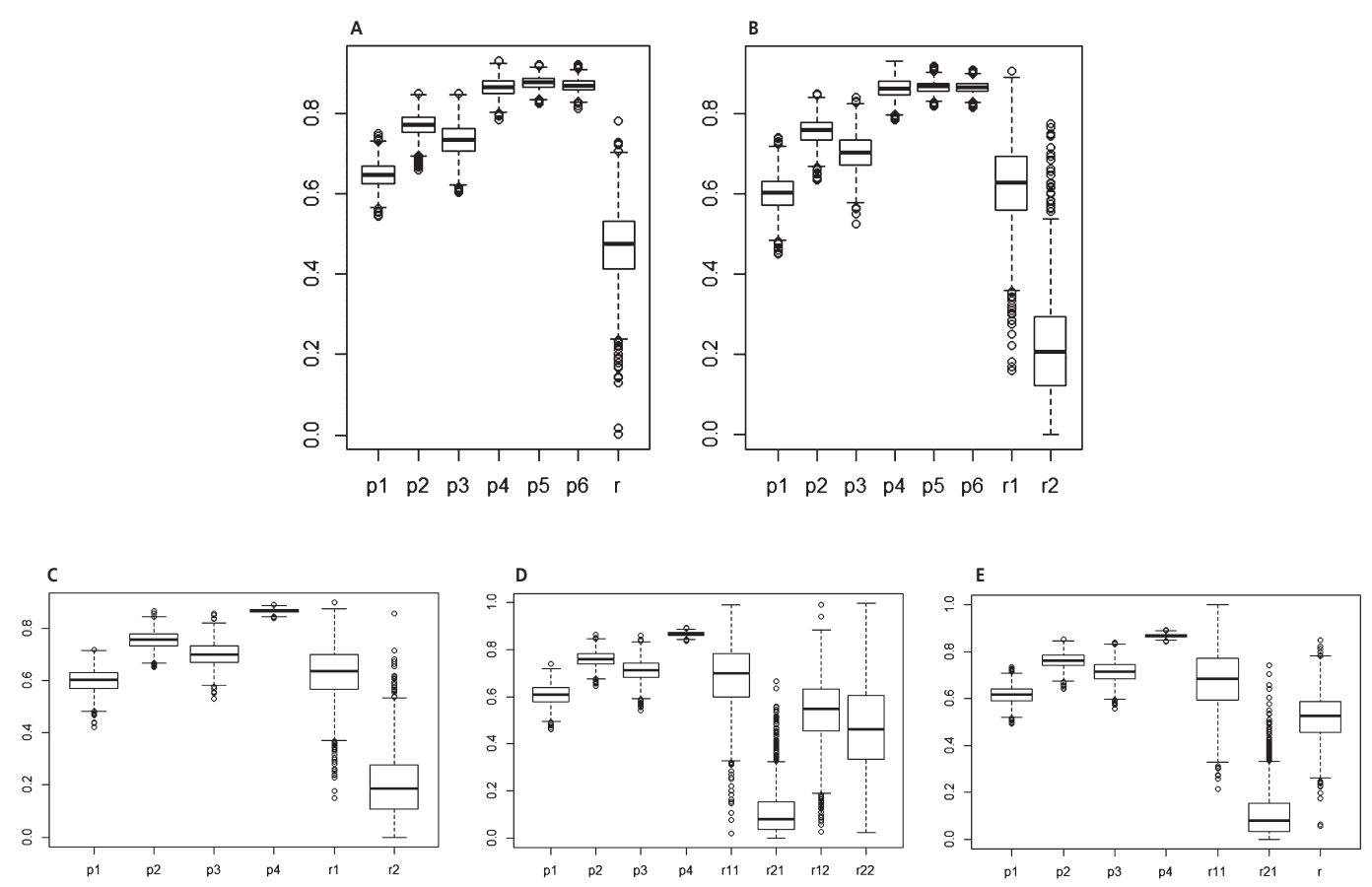

No entanto, os Modelos 1, 2 e 3 não levam em conta o fato de que alguns indivíduos experimentaram mobilidade ocupacional mais recentemente do que outros. A mobilidade ocupacional, diferentemente da de renda, ocorre principalmente no início da carreira. Para testar se há um efeito da mobilidade de classe no 
voto é importante levar em conta alguma medida que indique o tempo da mobilidade social. A melhor que temos, neste caso, é a idade das pessoas. Indivíduos mais velhos experimentaram mobilidade social há mais tempo do que indivíduos mais novos. De fato, a literatura mostra (Goldthorpe, 1980; De Graaf et al., 1995) que o efeito da classe de origem (da mobilidade social) tende a ser mais evidente entre os indivíduos mais novos, que chegaram à classe de destino mais recentemente. Os indivíduos mais velhos estão mais “aculturados" em suas classes de destino e tendem a ter um padrão de voto mais semelhante ao de suas classes de destino do que aos de suas classes de origem. Em outras palavras, o efeito da classe de origem seria mais evidente entre os indivíduos mais jovens do que entre os mais velhos.

Para testar esta ideia (hipótese da aculturação), estimamos mais dois modelos que incluem duas coortes de idade como variável interveniente e as mesmas quatro classes de origem e de destino usadas no Modelo 3. Assim, o Modelo 4 testa a hipótese de que os efeitos $r_{1}$ e $r_{2}$ do Modelo 3 são diferentes entre as coortes de idade mais nova e mais velha; enquanto o Modelo 5 testa a hipótese de que os efeitos $r_{1} \mathrm{e}$ $r_{2}$ do Modelo 3 são distintos para a coortes de idade mais nova (18 a 40 anos), mas indistintos $(r)$ para a coorte de idade mais velha (41 ou mais anos).

Para considerar as coortes de idade e pesos diferentes para mobilidade ascendente e descendente, o Modelo 4 adiciona ao Modelo 3 um índice para coorte de idade $k, \operatorname{com} k=1,2$ :

$$
\begin{array}{ll}
y_{i j k} \sim \operatorname{Binomial}\left(n_{i j k}, m_{i j k}\right) & \\
m_{i j k}=r_{1 k} p_{i}+\left(1-r_{1 k}\right) p_{j}, & \text { se } i \geq j e \\
m_{i j k}=r_{2 k} p_{i}+\left(1-r_{2 k}\right) p_{j}, & \text { se } i<j \operatorname{com} i, j=1, \cdots, c
\end{array}
$$

Os resultados do MCMC para os parâmetros do Modelo 4 também são apresentados na Tabela 3. As estatísticas de ajuste $($ DIC $=156,7$ e Preditiva $=51,89)$ indicam que o Modelo 4 não se ajusta melhor do que o anterior. No entanto, se observarmos as distribuições das densidades a posteriori dos parâmetros $r_{21}$ e $r_{22}$ (efeito das classes de origem para mobilidade ascendente e descendente para a coorte de idade mais velha), fica evidente que ambos são muito parecidos, o que não ocorre para a distribuição dos mesmos parâmetros $\left(r_{11}\right.$ e $\left.r_{12}\right)$ para a coorte de idade mais jovem. Esses últimos estão em posições assimétricas. De fato, representam o voto assimétrico em termos das mobilidades ascendente e descendente. Além disso, na coorte de idade mais velha as influências das classes de origens diminuem, ou seja, quanto maior a idade, maior a influência da posição de destino no voto, o que significa que as pessoas mais velhas talvez estejam mais aculturadas às suas classes de destino. 
Esses resultados encontrados no Modelo 4 (ver gráfico D da Figura 1) nos levaram a propor o Modelo 5. Este último testa a hipótese de que há efeitos assimétricos para mobilidade ascendente e descendente $\left(r_{11}\right.$ e $\left.r_{12}\right)$ para a coorte de idade mais nova, mas que não há assimetria para a coorte de idade mais velha. De acordo com a estatística DIC o Modelo $5($ DIC $=154,9)$ se ajusta melhor do que o Modelo $4($ DIC $=156,7)$, mas não é melhor do que o Modelo 3 (DIC $=90,13$ ). No entanto, se usarmos a preditiva o Modelo 5 (Preditiva $=48,7$ ), chegamos ao que melhor se ajusta aos dados. As evidências apresentadas na Figura 1 sugerem que a escolha do Modelo 5 como o melhor para descrever os dados faz sentido substantivamente e não é totalmente rejeitada estatisticamente. Nesse sentido, escolhemos o Modelo 5 como sendo o mais adequado.

Os parâmetros estimados pelo Modelo 5 nos permitem avaliar as hipóteses levantadas. Em primeiro lugar, os parâmetros para voto em Lula entre os indivíduos que não tiveram mobilidade intergeracional (parâmetros da diagonal) indicam claramente que membros imóveis das classes trabalhadoras (v+VI, viIa e virb) tenderam a votar significativamente mais em Lula do que as pessoas imóveis em todas as outras classes. Em contraste, indivíduos imóveis nas classes de profissionais e administradores (I+II) e na classe de pequenos proprietários (IV) tenderam a votar menos em Lula do que os membros imóveis das classes trabalhadoras. Os indivíduos imóveis na classe de trabalhadores não manuais de rotina ficaram em uma posição intermediária. A escolha do Modelo 5 como o preferido para esses dados sugere que não podemos rejeitar as hipóteses 3 e 5 .

\section{Discussão e conclusões}

Os modelos estatísticos, usando referência hierárquica sobre os componentes da diagonal da tabela de mobilidade social, conseguiram elucidar características importantes das preferências de voto declarado para a eleição de 2006. Além da associação entre classes trabalhadoras e voto no PT, os modelos estimados permitiram observar a influência da mobilidade ascendente e descendente no voto. A taxa de influência da origem quando a mobilidade é ascendente, $r_{l}$, é estatisticamente diferente da taxa de influência da origem quando a mobilidade é descendente, $r_{2}$. Sob o enfoque bayesiano, essa conclusão é obtida analisando as distribuições a posteriori dos dois parâmetros apresentados na Tabela 3 e na Figura 1.

O que chama atenção nos resultados é que o eleitor deu maior peso à posição de origem de classe no voto em Lula (PT) quando a mobilidade foi ascendente do que quando a mobilidade foi descendente. Isso pode indicar aprovação do governo do PT no mandato anterior (2002 a 2006), significando voto para reeleição de Lula daqueles que obtiveram mobilidade ascendente, mesmo não votando como as classes de ori- 
gem superiores. O mesmo efeito ocorre para os indivíduos que tiveram mobilidade descendente, só que na direção de não aprovação do governo. Tendo em vista que não há tendência de voto mais à direita para pessoas com mobilidade descendente e ascendente, devemos rejeitar a hipótese 4, mas não podemos rejeitar a hipótese 5 , que também prevê voto assimétrico, embora no sentido oposto ao inicialmente sugerido por Lipset e Bendix (1959).

Avançamos a análise ajustando modelos que levavam em conta a idade dos eleitores, que é um indicador do tempo em que as pessoas estão em uma determinada classe de destino. Esses modelos indicaram que o efeito assimétrico da mobilidade social está presente principalmente entre os mais jovens, que experimentaram mobilidade social mais recentemente. Isso significa que, conforme vimos, a melhor estratégia para analisar os dados por coortes de idade é utilizar modelos que separem os efeitos de mobilidades ascendente e descendente. A influência do tempo de socialização é evidente, uma vez que o efeito assimétrico está presente entre os mais jovens, mas não entre os mais velhos (Modelo 5). A principal diferença aparece na coorte de 18 a 40 anos, na qual a taxa de influência da origem para mobilidade ascendente é de 0,680 (média a posteriori de $r_{11}$ ), contra taxa de influência da origem para mobilidade descendente de 0,110 (média a posteriori de $r_{21}$ ). A diferença não é observada na coorte de idade mais velha, ou seja, não há diferença do efeito da origem entre as mobilidades descendente e ascendente.

Esses resultados são importantes para estabelecermos um diálogo com as cinco hipóteses que destacamos a partir da literatura sobre classe, mobilidade social e voto. A primeira hipótese é a do efeito de composição, que simplesmente sugere que a relação entre classe e voto depende dos níveis agregados de mobilidade social. Iniciamos nossas análises, ainda de forma descritiva, mostrando que de fato a relação entre voto e classe seria mais forte se não houvesse mobilidade social. Houve efeito de composição principalmente porque pessoas que entram nas classes de profissionais e administradores (I+II) e de pequenos proprietários (IV) tendiam a votar mais no PT do que as pessoas imóveis nestas classes.

A segunda hipótese, de que a preferência política das pessoas que experimentaram mobilidade social estaria mais próxima da preferência típica da sua classe de destino do que daquela mais comum em sua classe de origem $(\mathrm{H} 1)$, não se mostrou verdadeira para o caso brasileiro na eleição presidencial de 2006. Em todos os resultados, ocorreu uma ponderação entre a influência da origem e a do destino de classe ( $r$ próximo de 0,5). E, quando os modelos separaram as direções de mobilidade, a influência da origem foi mais acentuada para mobilidade ascendente e menos acentuada para mobilidade descendente. Assim, parece que o entendimento do voto naquela eleição não pode se limitar à "teoria econômica do voto". 
Nesse sentido, propusemos uma terceira hipótese: quanto mais velha a pessoa for, menor será o efeito da classe de origem em relação ao efeito da classe de destino. De fato, o modelo que melhor se ajustou aos dados (Modelo 5) indica que há efeito assimétrico da origem nos casos das mobilidades ascendente e descendente para a coorte de idade mais nova, mas não para a coorte de idade mais velha. Em outras palavras, nossas análises indicam que a hipótese de aculturação parece ser válida, na medida em que o tempo dentro de uma determinada classe de destino diminui o efeito da classe de origem.

A hipótese clássica do voto assimétrico (H4) não foi confirmada, tendo em vista que encontramos uma assimetria no sentido oposto ao previsto por Lipset e Bendix (1959). A assimetria que observamos para os mais jovens revela um padrão de adaptação diferente de acordo com a mobilidade descendente e a ascendente. Os Modelos 3 e 5 mostraram que o efeito da direção da mobilidade é estatisticamente significativo e, portanto, deve ser levado em consideração ao analisar a declaração de voto.

Os resultados dão margem para algumas interpretações do caso brasileiro. Nas eleições anteriores (2002), o voto em Lula (PT) não tinha o suporte de uma parte da classe trabalhadora e era associado a uma elite intelectual, como aparece em algumas análises empíricas (Carreirão, 2002; Limongi e Guarnieri, 2015). Nas eleições presidenciais de 2006 e 2010, houve uma mudança na base eleitoral do PT, seja referente à adesão de uma fração de classe como defende Singer (2012), seja como resultado de um "governo de sucesso", como sugerem Peixoto e Rennó (2011). O que deve ser destacado é que os modelos que estimamos neste artigo conseguem elucidar esse ponto de inflexão no eleitorado brasileiro. Fica evidente que há uma mudança no voto em Lula na coorte de idade de 18 a 40 anos. O voto bastante influenciado pela classe de origem no caso da mobilidade ascendente e o efeito contrário no caso de mobilidade descendente mostram que os mais jovens de alguma forma estão desalinhados do voto usual da classe da qual estão chegando. Mas esse desalinhamento favoreceu, em ambos os sentidos, o voto em Lula. Aqueles com mobilidade descendente tenderam a votar de forma mais parecida com sua classe de destino (mais PT); com mobilidade ascendente, de forma mais parecida com sua classe de origem (mais PT). No caso da mobilidade descendente, a classe de destino parece ter tido proeminência, o que pode indicar que, apesar da piora em termos de posição de classe, os eleitores permaneceram acreditando que o governo poderia melhorar sua situação. Ou seja, parece que essas pessoas votaram prospectivamente, esperando uma melhora no futuro. Em contraste, aqueles que experimentaram mobilidade ascendente permaneceram mais fiéis à sua classe de origem e votaram de acordo com seu padrão de socialização, o que pode indicar que a teoria dos grupos de referência é válida para explicar o padrão de voto nas eleições de 2006 ou, então, 
que essas pessoas votaram retrospectivamente, atribuindo sua mobilidade ascendente ao primeiro governo de Lula.

À primeira vista, nossos resultados levam a crer que tanto a teoria econômica do voto quanto a teoria dos grupos de referência parecem ser relevantes para explicar os padrões que encontramos. Em contrapartida, o fato de haver influência assimétrica da origem de classe nos leva a defender a validade da hipótese da aculturação de classe (Blau, 1956). Sugerimos que os estudiosos das eleições brasileiras, que em sua maioria parecem favorecer as teorias econômicas do voto, também levem em conta a perspectiva sociológica. Esperamos que nossos resultados contribuam tanto para estimular novas pesquisas na área de estudos eleitorais, testando criativamente a perspectiva sociológica, quanto para incentivar o uso de métodos bayesianos de análise de dados em ciências sociais.

\section{Referências Bibliográficas}

Alford, Robert R. (1962), "A suggested index of the association of social class and voting". Public Opinion Quarterly, 3 (26): 417-425. . (1963), Party and society: the Anglo-American democracies. Westport, Greenwood.

Blau, Peter M. (1956), "Social mobility and interpersonal relations". American Sociological Review, 21: 290-295.

Clark, Terry Nichols \& Lipset, Seymour Martin. (1991), “Are social classes dying?” International Sociology, 4(6): 347-410.

Clifford, Peter \& Heath, Anthony F. (1993), “The political consequences of social mobility”. Journal of the Royal Statistical Society, Series A, 156 (1): 51-61.

Carreirão, Yan de Souza. (2002), A decisão do voto nas eleições presidenciais brasileiras. Florianópolis/Rio de Janeiro, Editora da UFSC/Editora FGV.

Cogdon, Peter. (2005), Bayesian models for categorical data. Chichester, John Wiley \& Sons.

De GraAf, Nan Dirk et al. (1995), "Class mobility and political preferences: individual and contextual effect”. American Journal of Sociology, 4 (100): 997-1027.

Downs, Anthony. (1957), An economic theory of democracy. Nova York, Harper.

ERICKSON, Robert \& GOLDTHORPE, John H. (1993), The constant flux: a study of class mobility in industrial societies. Oxford, Oxford University Press.

Evans, Geoffrey. (1999), The end of class politics? Class voting in comparative context. Oxford, Oxford University Press.

Evans, Geoffrey \& De GraAf, Nan Dirk. (2013), Political choice matters: explaining the strength of class and religious cleavages in cross-national perspective. Oxford, Oxford University Press.

Gamerman, Dani \& Lopes, Hedibert F. (2006), Markov chain Monte Carlo: stochastic simulation for Bayesian inference. 2. ed. Boca Raton/Londres/Nova York, Chapman \& Hall/CRC. 
Gelman, Andrew et al. (2003), Bayesian data analysis. 2. ed. Boca Raton/Londres/Nova York/ Washington, Chapman \& Hall/crC.

Gill, Jeff. (2015), Bayesian methods: a social and behavioral science approach.3. ed. Boca Raton/ Londres /Nova York, Chapman \& Hall/CRC.

Goldthorpe, John H. (1980), Social mobility and class structure in modern Britain. Oxford, Clarendon.

Heath, Anthony Francis et al. (1985), How Britain votes. Oxford, Pergamon.

Hout, Mike et al. (1993), “The persistence of classes in post-industrial societies". International Sociology, 3 (8): 259-277.

Korpi, Walter. (1983). The democratic class struggle. Londres, Routledge \& Kegan Paul.

Limongi, Fernando \& Guarnieri, Fernando. (2015), "Competição partidária e voto nas eleições presidenciais no Brasil”. Opinião Pública, 1(21): 60-86.

Lipset, Seymour Martin \& BENdix, Reinhard. (1959), Social mobility in industrial society. Berkeley, University of California Press.

MARX, Karl. (1926). The eighteenth brumaire of Louis Bonaparte. Londres, Unwin.

Migon, Helio S. \& Gamerman, Dani. (1999), Statistical inference: an integrated approach. Londres, Arnold.

Migon, Helio S. et al. (2008), "Modelos hierárquicos e aplicações”. Minicurso do $18^{\circ}$ Sinape, São Pedro.

Nieuwbeerta, Paul. (1995), The democratic class struggle in twenty countries, 1945-1990. Amsterdã, Thesis Publishers.

Nieuwbeerta, Paul et al. (2000), "The effects of class mobility on class voting in post-war western industrialized countries”. European Sociological Review, 4 (16): 327-348.

Peixoto, Vitor \& Rennó, Lucio. (2011), “Mobilidade social ascendente e voto: as eleições presidenciais de 2010 no Brasil”. Opinião Pública, 2 (17): 304-332.

Ribeiro, Carlos Antonio Costa. (2007). Estrutura de classe e mobilidade social no Brasil. Bauru, Edusc. . (2009), Desigualdade de oportunidades no Brasil. Belo Horizonte, Argumentum.

Singer, André. (2012), Os sentidos do lulismo: reforma gradual e pacto conservador. São Paulo, Companhia das Letras.

SobeL, Michael E. (1981), "Diagonal mobility models: a substantively motivated class of designs for the analysis of mobility effects”. American Sociological Review, 6 (46): 893-906. 


\section{Resumo}

Voto assimétrico, classes e mobilidade social no Brasil

Este artigo analisa a relação entre a estrutura de classes, os padrões de mobilidade social dos eleitores e a declaração retrospectiva de voto no segundo turno da eleição presidencial de 2006. As análises permitem discutir a hipótese do voto assimétrico, segundo a qual a correlação entre classes sociais e voto é mediada pelos padrões de mobilidade intergeracional ascendente e descendente. Outras hipóteses correlatas também são discutidas a partir das análises empíricas baseadas na estimação de modelos hierárquicos bayesianos.

Palavras-chave: Classes sociais; Mobilidade social; Voto.

\section{Abstract}

Asimmetric vote, class and social mobility in Brazil

This paper analyzes the relationship among class structure, patterns of social mobility and voting at the Brazilian presidential election of 2006. The analysis aims to discuss and test the hypothesis of asymmetrical vote claiming that the correlation between social class and voting is mediated by the patterns of upward and downward intergenerational mobility. Other ancillary hypothesis are also discussed and empirically tested. All statistical estimations are done using Bayesian hierarchical models.

Keywords: Social class; Social mobility; Vote.

Texto recebido em 19/1/2016 e aprovado em 2/2/2016. DOI: 10.11606/0103-2070.ts.2016. 110049.

Carlos Antonio Costa Ribeiro é professor e pesquisador do Instituto de Estudos Sociais e Políticos (Iesp) da Universidade do Estado do Rio de Janeiro (Uerj) e PhD em sociologia pela Columbia University. Foi pesquisador convidado do Center for Advanced Study in the Behavioral Sciences (CASBS) da Stanford University. E-mail: carloscr@iesp.uerj.br.

Vinicius Pinheiro Israel é professor do Departamento de Métodos Estatísticos da UfrJ e doutorando em sociologia pelo IESP-UERJ, possui formação multidisciplinar, sendo graduado em matemática (UFRJ) e em ciências sociais (UERJ), com mestrado em métodos numéricos e doutorado em estatística, ambos pela UFRJ.E-mail: vinicius@im.ufrj.br. 


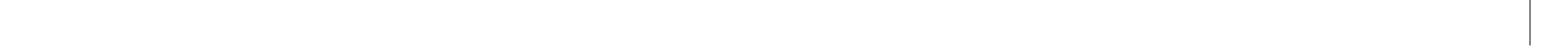

